\title{
Liver transplantation-Economics in the less developed world
}

\author{
Sanjiv Saigal $\cdot$ Sudeep R. Shah
}

Received: 5 December 2011 / Accepted: 12 December 2011 /Published online: 7 January 2012

(C) Indian Society of Gastroenterology 2012

Though liver transplant has been a well-established treatment for liver diseases in developed countries for several decades, developing countries have lagged behind in this field. However, in the last decade, there has been major progress in the field of liver transplantation in India. In particular, living-related liver transplant (LRLT) has flourished over the last five years [1]. Among developing countries, India currently performs the largest number of LRLTs and drains patients from some surrounding countries as well.

In the Western world, the government bears the expenditure on transplant programs. In some countries, insurance companies have recognized that it is more economical to fund liver transplantation than to pay for the management of end-stage liver disease, and now cover for this procedure. India spends much less on healthcare than the West. Communicable diseases such as tuberculosis and malaria take precedence, and few government hospitals would spare the budget to set up an LDLT program. The vast majority of liver transplants are thus performed in the private sector [1]. A certain section of the population is able to struggle and gather the money required for liver transplant. However, the expenditure does not end with surgery; rather, a successful procedure entails lifelong immunosuppression, a costly undertaking. Expectedly, the patients and their families are keen to know the magnitude of this expenditure in advance, and whether this cost burden can be somehow reduced. Fortunately for these patients, India is blessed with a strong

\section{S. Saigal}

Medanta, The Medicity,

Sector 38,

Gurgaon, Haryana 122 001, India

S. R. Shah $(\bowtie)$

P D Hinduja National Hospital and Medical Research Centre,

Mumbai 400 016, India

e-mail: shahsudeep@hotmail.com pharmaceutical industry that manufactures generic drugs [2]. These companies sell drugs such as tacrolimus and mycophenolate mofetil (MMF) at a fraction of the price in the developed world.

A paper by Sudhindran et al. [3] from Cochin in this issue of the Journal provides valuable information on the cost of immunosuppression over the first one-year period after LDLT in Indian patients. The most interesting revelation of this study perhaps was that the cost of calcineurin inhibitor (CNI) therapy in India is extremely low. Using a generic preparation, the cost of this treatment was in the range of Indian Rupees (INR) 3,000 to INR 5,000 per month ( $1 \mathrm{USD}=$ approximately INR 50 ). Interestingly, the largest component of the cost of CNI therapy was the expenditure on monitoring of drug levels. Using Abbott IMx kits, which are based on microparticle enzyme immunoassay technology, each measurement of tacrolimus drug level in their institution cost INR 3,900 which was roughly similar to the cost of onemonth supply of the CNI drug. The authors therefore suggest that it may be useful to find ways of tailoring downwards the need for monitoring of these levels in an attempt to reduce the cost of immunosuppressive treatment. However, the cost of drug monitoring that the authors have mentioned appears a bit high, since in many transplant centers in India such drug assay costs between INR 2,100 and INR 3,000 (personal communication).

It must be noted that, as a protocol, the authors of this current paper accepted a lower level of CNI during the first few months after LRLT. This is similar to the practice in other transplant centers. It has been observed that CNI drug levels needed to prevent rejection are lower in the LRLT setting than following deceased donor liver transplantation (DDLT) [4]. Further, the authors of the current paper were fairly liberal in the use of antimetabolites such as MMF in support.

The authors note that the blood levels produced by the generic CNI preparations used in the study were predictable 
and reproducible. These are reassuring data that lay to rest doubts about the efficacy of generic products. The original tacrolimus_-Prograf (Astellas, Japan) - is now available in India. However, the ready availability of low-price generics in India has ensured that this product is sold here at a competitive price, much lesser than that in other countries. Most generic brands available in India appear to derive the raw material from the same supplier; only the tablet manufacturing and packaging steps are performed independently by each company.

The rate of rejection of the transplanted livers was reported by the authors as low. This is in keeping with the experience of most transplant centers that the use of tacrolimus in appropriate doses ensures a rate of acute rejection below $15 \%$ to $20 \%$; further, hardly any graft is lost to chronic rejection in compliant patients.

Another point that arises from this cost audit is the burden inflicted by the use of MMF. This drug is far more expensive than the CNIs. Use of MMF has been shown to reduce the requirement of CNI drugs, a definite advantage in patients with a low glomerular filtration rate [5]. Such 'nephroprotection' is particularly required in sick patients with an elevated baseline creatinine. Some patients with normal serum creatinine may have a deceptively low GFR as creatinine production itself is impaired in patients with liver disease because of extremely poor muscle mass. The authors suggest that azathioprine may be used to augment the effect of CNI instead of MMF to reduce costs. This turns the wheel through a full circle. Azathioprine was the main immunosuppressant in the pre-CNI era; however, its use was associated with a high rate of rejection of transplanted organs and subsequent graft loss. Once CNIs were shown to be superior and were established as the primary immunosuppressive drugs, azathioprine became an adjunct. Subsequently, MMF was marketed as a product with superior efficacy to azathioprine. However, the trial, which led to the approval for MMF, used doses up to $3 \mathrm{~g} /$ day [6]. Such doses are exceedingly difficult to administer due to debilitating gastrointestinal adverse effects such as cramps and diarrhea, and a smaller dose of 1-2 g/day is currently used. However, it is unclear whether MMF in these lower doses is more efficacious than azathioprine [7]. If there is no difference in efficacy, it would be worthwhile using azathioprine in place of low-dose MMF, particularly in low-income countries such as India. Unfortunately, the pharmaceutical industry is unlikely to be interested in a clinical trial comparing MMF and azathioprine, since the latter, being past its patent expiry period, is extremely cheap.

Most Indian centers discontinue MMF one to two years after liver transplant and use tacrolimus monotherapy in those patients who are stable. In fact, in some stable patients with good renal function, it may be possible to use monotherapy even within the first year.

The study by Sudhindaran et al. should be sweet news for Indian patients needing LRLT and for Indian transplant surgeons alike. The authors showed that tacrolimus monotherapy using generic preparations was cheap while being effective, and that half their patients could be maintained on tacrolimus monotherapy by the end of the first year postLDLT. Thus, in a large proportion of Indian patients, maintaining the recently acquired, expensive liver graft may not be so unaffordable in the long run, costing only INR 3,000 to INR 5,000 per month - comparable to the cost of treating insulin-dependent diabetes mellitus.

\section{References}

1. Varma V, Mehta N, Nundy S. Liver Transplantation in India. In: Nundy S, Soin AS, (eds.) ECAB Clinical Update: Liver Transplantation. New Delhi: Elsevier; 2011;pp. 8-14.

2. Love J. The production of generic drugs in India. Br Med J. 2011:342:d1694.

3. Sudhindran S, Aboobacker S, Menon RN, Unnikrishnan G, Sudheer $\mathrm{OV}$, Dhar P. Cost and efficacy of immunosuppression using generic products following living donor liver transplantation in India. Indian J Gastroenterol. 2012;31. doi:10.1007/s12664-011-0138-0.

4. Jain A, Venkataramanan R, Sharma R, et al. Pharmacokinetics of tacrolimus in living donor liver transplant and deceased donor liver transplant recipients. Transplantation. 2008;85:554-60.

5. Jain A, Vekatramanan R, Eghtesad B, et al. Long-term outcome of adding mycophenolate mofetil to tacrolimus for nephrotoxicity following liver transplantation. Transplantation. 2005;80:859-64.

6. Wiesner R, Rabkin J, Klintmalm G, et al. A randomized doubleblind comparative study of mycophenolate mofetil and azathioprine in combination with cyclosporine and corticosteroids in primary liver transplant recipients. Liver Transpl. 2001;7:442-50.

7. Germani G, Pleguezuelo M, Villamil F, et al. Azathioprine in liver transplantation: a reevaluation of its use and a comparison with mycophenolate mofetil. Am J Transplant. 2009;9:1725-31. 